\title{
The Young Measure Representation for Weak Cluster Points of Sequences in $M$-spaces of Measurable Functions \\ by
}

Hôǹg Thái NGUYỄ̃ and Dariusz PĄCZKA

Presented by Czesław OLECH

Summary. Let $\langle X, Y\rangle$ be a duality pair of $M$-spaces $X, Y$ of measurable functions from $\Omega \subset \mathbb{R}^{n}$ into $\mathbb{R}^{d}$. The paper deals with $Y$-weak cluster points $\bar{\phi}$ of the sequence $\phi\left(\cdot, z_{j}(\cdot)\right)$ in $X$, where $z_{j}: \Omega \rightarrow \mathbb{R}^{m}$ is measurable for $j \in \mathbb{N}$ and $\phi: \Omega \times \mathbb{R}^{m} \rightarrow \mathbb{R}^{d}$ is a Carathéodory function. We obtain general sufficient conditions, under which, for some negligible set $A_{\phi}$, the integral $I\left(\phi, \nu_{x}\right):=\int_{\mathbb{R}^{m}} \phi(x, \lambda) d \nu_{x}(\lambda)$ exists for $x \in \Omega \backslash A_{\phi}$ and $\bar{\phi}(x)=I\left(\phi, \nu_{x}\right)$ on $\Omega \backslash A_{\phi}$, where $\nu=\left\{\nu_{x}\right\}_{x \in \Omega}$ is a measurable-dependent family of Radon probability measures on $\mathbb{R}^{m}$.

1. Notations and some basic facts on Young measures. Let $\mu$ denote a complete separable $\sigma$-finite $\sigma$-additive positive measure on a $\sigma$-algebra $\mathfrak{A}$ of subsets of a set $\Omega$. Measurability will always mean $\mathfrak{A}$-measurability. Let $E$ be a separable Banach space. We will denote by $L^{\infty}(\Omega, E ; \mu)$, or briefly $L^{\infty}(E)$, the Banach space (of all equivalence classes) of essential $E$-norm-bounded measurable functions $u: \Omega \rightarrow E$ with norm $\|u\|_{L^{\infty}}:=$ $\operatorname{ess}_{\sup } \in \Omega\|u(x)\|_{E}$. Let $L^{1}(\Omega, E ; \mu)$, or briefly $L^{1}(E)$, denote the BochnerLebesgue space (of all equivalence classes) of $\mu$-integrable strongly measurable functions from $\Omega$ into $E$.

Let $\mathcal{M}\left(\mathbb{R}^{m}\right)$ be the Banach space of bounded signed Radon measures on $\mathbb{R}^{m}$ and $C_{0}\left(\mathbb{R}^{m}\right)$ be the Banach space of all continuous functions $f: \mathbb{R}^{m} \rightarrow \mathbb{R}$ with $\lim _{|\lambda| \rightarrow \infty} f(\lambda)=0$ equipped with the sup-norm, where $|\cdot|$ denotes the Euclidean norm in $\mathbb{R}^{m}$. It is known that $\left(C_{0}\left(\mathbb{R}^{m}\right)\right)^{*} \cong \mathcal{M}\left(\mathbb{R}^{m}\right)$. Let $L_{\omega}^{\infty}\left(\mathcal{M}\left(\mathbb{R}^{m}\right)\right.$ ) denote the Banach space (of all equivalence classes) of $C_{0}\left(\mathbb{R}^{m}\right)$ -

2000 Mathematics Subject Classification: 28A33, 46E27, 46E30, 46E40, 47H30.

Key words and phrases: Young measure representation, weak cluster points of sequences in non- $L^{p}$-type spaces of measurable functions, $M$-spaces, Banach lattices and non-solid generalized Orlicz spaces, Köthe-Bochner spaces. 
weakly measurable functions $\nu: \Omega \rightarrow \mathcal{M}\left(\mathbb{R}^{m}\right)$ with norm $\|\nu\|_{\infty}:=\| x \mapsto$ $\left|\nu_{x}\right|\left(\mathbb{R}^{m}\right) \|_{L^{\infty}}<\infty$, where $\left|\nu_{x}\right|\left(\mathbb{R}^{m}\right)$ is the total variation of $\nu_{x}$ on $\mathbb{R}^{m}$ and, for abbreviation, we write $L^{\infty}\left(\right.$ resp. $\left.\nu_{x}\right)$ instead of $L^{\infty}(\mathbb{R})($ resp. $\nu(x))$. It is known that $L_{\omega}^{\infty}\left(\mathcal{M}\left(\mathbb{R}^{m}\right)\right)$ can be interpreted as dual space $\left(L^{1}\left(C_{0}\left(\mathbb{R}^{m}\right)\right)\right)^{*}$ via the injection $\nu \mapsto\langle\cdot, \nu\rangle_{\mu}$, where $\langle h, \nu\rangle_{\mu}:=\int_{\Omega}\langle v(x), h(x)\rangle d \mu(x)$ for all $h \in L^{1}\left(C_{0}\left(\mathbb{R}^{m}\right)\right)$. Given a measurable function $z: \Omega \rightarrow \mathbb{R}^{m}$, define the parametrized Dirac measure $\delta_{z} \in L_{\omega}^{\infty}\left(\mathcal{M}\left(\mathbb{R}^{m}\right)\right)$ by

$$
x \in \Omega \mapsto \delta_{z}(x):=\delta_{z(x)} \quad \text { (the Dirac measure supported at } z(x) \text { ). }
$$

An element $\nu \in L_{\omega}^{\infty}\left(\mathcal{M}\left(\mathbb{R}^{m}\right)\right)$ is called a Young parametrized measure if $\nu_{x}\left(\mathbb{R}^{m}\right)=1 \mu$-a.e. Define $(\phi \circ z)(x):=\phi(x, z(x))$. A function $f: \Omega \times \mathbb{R}^{m} \rightarrow E$ is said to be Carathéodory if $f(\cdot, u)$ is measurable for every $u \in \mathbb{R}^{m}$ and $f(x, \cdot)$ is continuous for almost all $x \in \Omega$.

The formulations and proofs of the main results of the present paper are based on the following fundamental theorem $[2,3]$ about the Young measure representation in case of the pair $\langle X, Y\rangle=\left\langle L^{1}(\mathbb{R}), L^{\infty}(\mathbb{R})\right\rangle$ (see Theorem 1.1; cf. [20, p. 98-100], [8, Section 8.1, pp. 518-525], [5, 21]).

Theorem 1.1 (The Young measure representation; Ball [3], Balder [2]). Suppose that a sequence of measurable functions $z_{j}: \Omega \rightarrow \mathbb{R}^{m}$ satisfies the global tightness condition with respect to $\mu$ :

$$
\lim _{L \rightarrow \infty} \sup _{j \in \mathbb{N}} \mu\left\{x \in \Omega:\left|z_{j}(x)\right| \geq L\right\}=0 .
$$

Then there exist a subsequence $z_{j_{k}}$ and a Young measure $\nu=\left\{\nu_{x}\right\}_{x \in \Omega}$ such that $\delta_{z_{j_{k}}}$ is $L^{1}\left(C_{0}\left(\mathbb{R}^{m}\right)\right)$-weakly convergent to $\nu$ in $L_{\omega}^{\infty}\left(\mathcal{M}\left(\mathbb{R}^{m}\right)\right)$. Moreover, given a Carathéodory function $\psi: \Omega \times \mathbb{R}^{m} \rightarrow \mathbb{R}$, the following statements hold.

(Y1) If $\psi \circ z_{j_{k}}$ is $L^{\infty}(\mathbb{R})$-weakly convergent to $\bar{\psi}$ in $L^{1}(\mathbb{R})$, then, for some $\mu$-negligible set $A_{\psi} \in \mathfrak{A}$, the integral $\int_{\mathbb{R}^{m}} \psi(x, \lambda) d \nu_{x}(\lambda) \in \mathbb{R}$ exists for $x \in \Omega \backslash A_{\psi}$ and

$$
\bar{\psi}(x)=\int_{\mathbb{R}^{m}} \psi(x, \lambda) d \nu_{x}(\lambda) \quad \text { on } \Omega \backslash A_{\psi} .
$$

(Y2) If $\psi \circ z_{j_{k}}$ is sequentially $L^{\infty}(\mathbb{R})$-weakly pre-compact in $L^{1}(\mathbb{R})$, then, for some $\mu$-negligible set $A_{\psi} \in \mathfrak{A}$, the integral $I\left(\psi, \nu_{x}\right):=\int_{\mathbb{R}^{m}} \psi(x, \lambda) d \nu_{x}(\lambda)$ $\in \mathbb{R}$ exists for all $x \in \Omega \backslash A_{\psi}$ and $\psi \circ z_{j_{k}}$ is $L^{\infty}(\mathbb{R})$-weakly convergent to $\widetilde{\psi} \in L^{1}(\mathbb{R})$, where $\widetilde{\psi}(x):=I\left(\psi, \nu_{x}\right)$ for $x \in \Omega \backslash A_{\psi}$ and $\widetilde{\psi}(x):=0$ otherwise.

The generalization of Theorem 1.1 for the $L^{\Psi^{*}}(\mathbb{R})$-weak limit of $\tau \circ z_{j_{k}}$ in the Orlicz space $L^{\Psi}(\mathbb{R})$ is proved by P. Málek et al. [11, Th. 4.2.1, pp. 171$176]$ in the case when $\Psi$ and $\Psi^{*}$ are complementary non-power Orlicz functions, $\Psi$ satisfies the $\triangle_{2}$-condition [12], and $\tau: \mathbb{R}^{m} \rightarrow \mathbb{R}$ is continuous. 
2. Formulation of results. A linear space $Z \subset L^{0}\left(\mathbb{R}^{m}\right)$ is called an $M$-space if the inclusions $z \in Z$ and $\alpha \in L^{\infty}(\mathbb{R})$ imply that $\alpha z \in Z[16,18]$. If $m=1$ then it is easy to check that $M$-spaces $Z$ are just vector lattices. The Köthe associate space $Z^{\prime}$ with respect to $\mu$ of an $M$-space $Z$ is defined e.g. in [7, 9] for $m=1$, and in [15, 14, 18] for $m \geq 2$. By [16, Theorem 3.1], equivalently in case $m \geq 2, Z^{\prime}$ is defined by

$$
Z^{\prime}=\left\{z^{\prime} \in L^{0}\left(\mathbb{R}^{m}\right): z^{\prime}(x) \in \operatorname{vsupp} Z(x) \mu \text {-a.e., }\left\langle z, z^{\prime}\right\rangle_{\mu} \in \mathbb{R}, \forall z \in Z\right\} .
$$

Here $\left\langle z, z^{\prime}\right\rangle_{\mu}:=\int_{\Omega}\left(z(x), z^{\prime}(x)\right) d \mu(x)$, where $(\cdot, \cdot)$ denotes the Euclidean scalar product on $\mathbb{R}^{m}$, and the so-called vector support vsupp $Z$ can be equivalently defined by

$$
\operatorname{vsupp} Z(x):=\overline{\left\{z_{1}(x), z_{2}(x), \ldots\right\}} \quad \mu \text {-a.e. }
$$

for some sequence $z_{n} \in Z$ such that $z \in Z \Rightarrow z(x) \in \overline{\left\{z_{1}(x), z_{2}(x), \ldots\right\}} \mu$-a.e. If $Z, Y \subset L^{0}\left(\mathbb{R}^{m}\right)$ are $M$-spaces and $Y \subset Z^{\prime}$, then $\langle Z, Y\rangle$ is a duality pair with respect to $\left\langle z, z^{\prime}\right\rangle_{\mu}\left(z \in Z, z^{\prime} \in Y\right)$, and we write $\langle Z, Y\rangle_{\mu}$.

Let $\langle Z, Y\rangle$ be a duality pair of vector spaces. A set $\mathcal{N} \subset Z$ is called sequentially $Y$-weakly pre-compact in $Z$ (or conditionally sequentially $Y$-weakly compact in $Z$ ) if each sequence $z_{j} \in \mathcal{N}$ has some $Y$-weak Cauchy subsequence $z_{j(k)}$. The space $Z$ is called sequentially $Y$-weakly complete if each $Y$-weak Cauchy sequence is $Y$-weakly convergent in $Z$.

Theorem 2.1. Let $X, Y \subset L^{0}\left(\mathbb{R}^{d}\right)$ be $M$-spaces, supp $X=\Omega$, vsupp $X(x)$ $=\operatorname{vsupp} Y(x) \mu$-a.e., and $Y \subset X^{\prime}$, where $X^{\prime}$ is the Köthe associate space of $X$ with respect to $\mu$. Suppose that a sequence $z_{j} \in L^{0}\left(\mathbb{R}^{m}\right)$ satisfies (GB) with respect to $\mu$, and a Carathéodory function $\phi: \Omega \times \mathbb{R}^{m} \rightarrow \mathbb{R}^{d}$ satisfies $\phi\left(x, \mathbb{R}^{m}\right) \subset \operatorname{vsupp} X(x) \mu$-a.e. Moreover, let $z_{j_{k}}$ and $\nu$ be as in Theorem 1.1. Then the following statements hold.

(Y3) If $\phi \circ z_{j_{k}}$ is $Y$-weakly convergent to $\bar{\phi}$ in $X$, then, for some $\mu$-negligible set $A_{\phi} \in \mathfrak{A}$, the integral $\int_{\mathbb{R}^{m}} \phi(x, \lambda) d \nu_{x}(\lambda)$ exists in $\operatorname{vsupp} X(x)$ for $x \in \Omega \backslash A_{\phi}$ and

$$
\bar{\phi}(x)=\int_{\mathbb{R}^{m}} \phi(x, \lambda) d \nu_{x}(\lambda) \quad \text { on } \Omega \backslash A_{\phi} .
$$

(Y4) If $X=Y^{\prime}$ and $\phi \circ z_{j_{k}}$ is sequentially $Y$-weakly pre-compact in $X$, then, for some $\mu$-negligible set $A_{\phi} \in \mathfrak{A}$, the integral $I\left(\phi, \nu_{x}\right):=$ $\int_{\mathbb{R}^{m}} \phi(x, \lambda) d \nu_{x}(\lambda)$ exists in $\operatorname{vsupp} X(x)$ for all $x \in \Omega \backslash A_{\phi}$ and $\phi \circ z_{j_{k}}$ is $Y$-weakly convergent to $\widetilde{\phi}$ in $X$, where $\widetilde{\phi}(x):=I\left(\phi, \nu_{x}\right)$ for $x \in \Omega \backslash A_{\phi}$ and $\widetilde{\phi}(x):=0$.

Condition 2.2 (Local tightness condition, [11, p. 171], [20]). A sequence $z_{j} \in L^{0}\left(\mathbb{R}^{m}\right)$ satisfies 


$$
\lim _{L \rightarrow \infty} \sup _{j \in \mathbb{N}} \mu\left\{x \in C_{q}:\left|z_{j}(x)\right| \geq L\right\}=0 \quad(\forall q \in \mathbb{N})
$$

for a nondecreasing sequence $C_{q} \in \mathfrak{A}$ with $\mu\left(C_{q}\right)<\infty$ and $\bigcup_{q \in \mathbb{N}} C_{q}=\Omega$.

Theorem 2.3. Let $\mu(\Omega)=\infty$ and let $X, Y$ and $\phi$ be as in Theorem 2.1. If a sequence $z_{j} \in L^{0}\left(\mathbb{R}^{m}\right)$ satisfies $(\mathrm{LB})$ with respect to $\mu$, then the statements (Y3)-(Y4) of Theorem 2.1 remain true.

A normed space $Z \subset L^{0}\left(\mathbb{R}^{m}\right)$ with norm $\|\cdot\|_{Z}$ is called a normed $M$ space if the inclusions $z \in Z$ and $\alpha \in L^{\infty}(\mathbb{R})$ imply that $\alpha z \in Z$ and $\|\alpha z\|_{Z} \leq\|\alpha\|_{L^{\infty}}\|z\|_{Z}[16,18]$. The regular part $Z^{\circ}$ of a normed $M$-space $Z$ is defined to be the normed $M$-subspace of all elements $z \in Z$ satisfying $\lim _{\mu_{*}(D) \rightarrow 0}\left\|\chi_{D} z\right\|_{Z}=0$, where $\mu_{*}:=\mu$ if $\mu(\Omega)<\infty$ and $\mu_{*}$ is a fixed finite positive measure equivalent to $\mu$ if $\mu(\Omega)=\infty$, and $\chi_{D}$ denotes the characteristic function of $D \in \mathfrak{A}$.

Proposition 2.4. Let $X, Y \subset L^{0}\left(\mathbb{R}^{d}\right)$ be $M$-spaces with $X \subset Y^{\prime}$, where $Y^{\prime}$ is the Köthe associate space of $Y$ with respect to $\mu$. Suppose that a sequence $z_{j} \in L^{0}\left(\mathbb{R}^{m}\right)$ and a Carathéodory function $\phi: \Omega \times \mathbb{R}^{m} \rightarrow \mathbb{R}^{d}$ satisfy one of the following conditions:

(SC1) There exist nondecreasing continuous functions $g, \gamma:[0, \infty) \rightarrow[0, \infty)$ such that

(a) $\lim _{t \rightarrow \infty} g(t)=\infty$ and $\lim _{t \rightarrow \infty} \gamma(t) / g(t)=0$;

(b) $\left\{\left(g \circ\left|z_{j}\right|\right) u_{0}\right\}_{j \in \mathbb{N}}$ is $Y$-weakly bounded in $X$, where $u_{0}: \Omega \rightarrow(0, \infty)$ is measurable, $u_{0} Y \subset L^{1}\left(\mathbb{R}^{d}\right)$, and $\operatorname{vsupp} X(x)=\operatorname{vsupp} Y(x)$ $\mu$-a.e.;

(c) $|\phi(x, \lambda)| \leq \gamma(|\lambda|) u_{0}(x)$ for $\mu$-almost all $x \in \Omega$ and all $\lambda \in \mathbb{R}^{m}$;

(SC2) There exists a Banach $M$-space $\Gamma$ with $Y \subset \Gamma^{\circ},\left(\Gamma^{\circ}\right)^{\prime} \subset X$ and

$$
\sup _{j \in \mathbb{N}}\left\|\phi \circ z_{j}\right\|_{\left(\Gamma^{\circ}\right)^{\prime}}<\infty \text {. }
$$

Then the sequence $\phi \circ z_{j}$ is sequentially $Y$-weakly pre-compact in $X$.

Remark 2.5. Proposition 2.4/(SC1) is a generalization of [20, Proposition 6.5] (where $Y=L^{1}(\mathbb{R})$ with $\mu(\Omega)<\infty$ ).

In the case of $\phi: \Omega \times \mathbb{R}^{m} \rightarrow E$ with $\operatorname{dim} E=\infty$, results analogous to Theorems 2.1 and 2.3 can be proved but only for a pair $\langle X, Y\rangle$ of Köthe-Bochner spaces $X, Y$ of $E$ - $/ E^{*}$-valued functions (see Theorem 2.6). Given a separable Banach space $E$ and a vector lattice $K \subset L^{0}(\mathbb{R})$, the Köthe-Bochner space $K(E)$ is defined as the space (of equivalence classes) of strongly measurable $E$-valued functions $z$ such that $\|z(\cdot)\|_{E} \in K$.

TheOREM 2.6. Let $K, \widetilde{K} \subset L^{0}(\mathbb{R})$ be vector lattices, $E$ be a Banach space and $E^{*}$ be its dual. Assume that: 
(a) $\operatorname{supp} K=\operatorname{supp} \widetilde{K}=\Omega$ and $\widetilde{K} \subset K^{\prime}$, where $K^{\prime}$ is the Köthe associate space of $K$ with respect to $\mu$;

(b) $E$ is separable and reflexive with $\operatorname{dim} E=\infty$.

If $\phi: \Omega \times \mathbb{R}^{m} \rightarrow E$ is a Carathéodory function and a sequence $z_{j} \in L^{0}\left(\mathbb{R}^{m}\right)$ satisfies either (GB) or (LB), then the statements (Y3)-(Y4) of Theorem 2.1 remain true for the Köthe-Bochner spaces $X=K(E)$ and $Y=\widetilde{K}\left(E^{*}\right)$ provided (2.1) (resp. $\widetilde{\phi}$ ) is substituted by

$$
\bar{\phi}(x)=(P)-\int_{\mathbb{R}^{m}} \phi(x, \lambda) d \nu_{x}(\lambda) \quad \text { on } \Omega \backslash A_{\phi}
$$

(resp. $\widetilde{\phi}(x)=(P)-\int_{\mathbb{R}^{m}} \phi(x, \lambda) d \nu_{x}(\lambda)$ for $\left.x \in \Omega \backslash A_{\phi}\right)$, where, for $x \in \Omega \backslash A_{\phi}$, the above integral exists as the Pettis integral of the function $\phi(x, \cdot): \mathbb{R}^{m} \rightarrow$ $E$ with respect to the measure $\nu_{x}$.

Proposition 2.7. Let $z_{j} \in L^{0}\left(\mathbb{R}^{m}\right)(j \in \mathbb{N})$. Then $(\mathrm{LB})$ with respect to $\mu$ follows from the condition:

$(\mathrm{LK})$ For $q \in \mathbb{N}$ there exist a normed lattice with monotone norm $K(q) \subset$ $L^{0}(\mathbb{R})$ and a continuous nondecreasing function $g_{q}:[0, \infty) \rightarrow[0, \infty)$ such that $\lim _{t \rightarrow \infty} g_{q}(t)=\infty$ and $\sup _{j \in \mathbb{N}}\left\|\chi_{C_{q}} g_{q}\left(\left|z_{j}(\cdot)\right|\right)\right\|_{K(q)}<\infty$ for a nondecreasing sequence $C_{q} \in \mathfrak{A}$ with $\mu\left(C_{q}\right)<\infty$ and $\bigcup_{q \in \mathbb{N}} C_{q}=\Omega$.

REMARK 2.8. Proposition 2.7 is an extension of the statement in [3, Remark 1, p. 209] (where $\left.K(q)=L^{1}(\mathbb{R})\right)$.

Remark 2.9. If $Z \subset L^{0}\left(\mathbb{R}^{m}\right)$ is a normed $M$-space and $\sup _{j \in \mathbb{N}}\left\|z_{j}\right\|_{Z}$ $<\infty$, then (LB) holds. Indeed, by [9, Corollary of Theorem IV.3.1], [23] $(m=1)$ and [16, Theorem 2.1/(3)] $(m \geq 2)$, the sequence $z_{j}$ is bounded in $L^{0}\left(\mathbb{R}^{m}\right)$ equipped with the quasi-norm $\|z\|_{L^{0}\left(\mathbb{R}^{m}\right)}:=\int_{\Omega} \frac{|z(x)|}{1+|z(x)|} d \mu_{*}(x)$. Hence, by [9, Section III.1.3-III.1.4], this sequence is bounded in $\mu$ on any $C_{q}$, and so (LB) follows. In particular, $Z$ can be assumed to be either a Banach lattice of scalar-valued functions (a solid space) or a non-solid generalized Orlicz space (see, e.g., $[1,12,17])$ of $\mathbb{R}^{m}$-valued functions with $m \geq 2$.

\section{Proofs of results of Section 2}

Proof of Theorem 2.1. We divide this proof into Steps 3.1-3.2.

SteP 3.1 (Proof of (Y3)). Given $y \in Y$, define $\phi_{y}: \Omega \times \mathbb{R}^{m} \rightarrow \mathbb{R}$ by $\phi_{y}(x, \lambda):=(y(x), \phi(x, \lambda))$. As $Y$ is an $M$-space we have $\alpha y \in Y$ for every $\alpha \in L^{\infty}(\mathbb{R})$, and from $Y \subset X^{\prime}$ we infer that

$$
\left\langle\phi \circ z_{j_{k}}, \alpha y\right\rangle_{\mu}=\left\langle\phi_{y} \circ z_{j_{k}}, \alpha\right\rangle_{\mu} \in \mathbb{R} .
$$

By Theorem 1.1/(Y2) for $\phi_{y}$ together with the assumption for $\phi \circ z_{j_{k}}$, we 
deduce that

$$
\left\langle\phi \circ z_{j_{k}}, \alpha y\right\rangle_{\mu}=\left\langle\phi_{y} \circ z_{j_{k}}, \alpha\right\rangle_{\mu} \rightarrow\langle\bar{\phi}, \alpha y\rangle_{\mu}=\left\langle\widetilde{\phi}_{y}, \alpha\right\rangle_{\mu} \in \mathbb{R}
$$

for all $\alpha \in L^{\infty}(\mathbb{R})$, where, for some $\widetilde{D}_{\phi y} \in \mathfrak{A}$ with $\mu\left(\Omega \backslash \widetilde{D}_{\phi y}\right)=0$, the integral $\int_{\mathbb{R}^{m}} \phi_{y}(x, \lambda) d \nu_{x}(\lambda) \in \mathbb{R}$ exists for $x \in \widetilde{D}_{\phi y}$, and $\widetilde{\phi}_{y}(x):=\int_{\mathbb{R}^{m}} \phi_{y}(x, \lambda) d \nu_{x}(\lambda)$ for $x \in \widetilde{D}_{\phi y}$ and $\widetilde{\phi}_{y}(x):=0$ otherwise. Hence,

$$
\begin{aligned}
\left\langle\bar{\phi}, \chi_{D} y\right\rangle_{\mu} & =\left\langle\widetilde{\phi}_{y}, \chi_{D}\right\rangle_{\mu} \\
& =\int_{D}\left[\int_{\mathbb{R}^{m}}(y(x), \phi(x, \lambda)) d \nu_{x}(\lambda)\right] d \mu(x) \in \mathbb{R} \quad\left(D \in \mathfrak{A}, D \subset \widetilde{D}_{\phi y}\right) .
\end{aligned}
$$

On the other hand, $\left\langle\bar{\phi}, \chi_{D} y\right\rangle_{\mu}=\int_{D}(y(x), \bar{\phi}(x)) d \mu(x)$ for any $D \in \mathfrak{A}$ with $D \subseteq \widetilde{D}_{\phi y}$. By the Radon-Nikodym theorem, we deduce that for $y \in Y$ there exists $D_{\phi y} \in \mathfrak{A}$ such that $D_{\phi y} \subset \widetilde{D}_{\phi y}, \mu\left(\widetilde{D}_{\phi y} \backslash D_{\phi y}\right)=0$, and

$$
(y(x), \bar{\phi}(x))=\int_{\mathbb{R}^{m}}(y(x), \phi(x, \lambda)) d \nu_{x}(\lambda) \in \mathbb{R} \quad\left(\forall x \in D_{\phi y}\right) .
$$

Now, we consider $X \subset L^{0}\left(\Omega, \mathbb{R}^{d}\right)$ and $Y \subset X^{\prime}$ for $d>1$ (the case $d=1$ can be handled analogously upon using [9, Corollary IV.3.2] for $\operatorname{supp} Y=$ $\operatorname{supp} X=\Omega$ ). By [16, Theorem 3.1], there exists a sequence of representative families $G_{q}=\left\{u_{1 q}, \ldots, u_{d q}\right\}$ of the $M$-space $Y$ such that the sets $\operatorname{supp} G_{q}$ $\in \mathfrak{A}$ are mutually disjoint, and

(1) $\mu\left(\operatorname{supp} Y \backslash \bigcup_{q=1}^{\infty} \operatorname{supp} G_{q}\right)=0$;

(2) $\left|u_{1 q}(x)\right|=\cdots=\left|u_{d(q) q}(x)\right|=1$ and $\left|u_{i q}(x)\right|=0(i \notin\{1, \ldots, d(q)\})$ for $x \in \operatorname{supp} G_{q}$ and $d(q)=\operatorname{dim} \operatorname{vsupp} Y(x)$ on $\operatorname{supp} G_{q}$.

By the definition [16] of the representative family $G_{q}$, we have $u_{i q} \in Y$ and the linear hull of $\left\{u_{1 q}(x), \ldots, u_{d q}(x)\right\}$ coincides with vsupp $Y(x)$ for $x \in \operatorname{supp} G_{q}$. Hence, by (3.1), for $\chi_{\operatorname{supp} G_{q}} u_{p q} \in Y(1 \leq p \leq d(q))$ there exists $D_{p q} \in \mathfrak{A}$ such that $D_{p q} \subset \operatorname{supp} G_{q}, \mu\left(\operatorname{supp} G_{q} \backslash D_{p q}\right)=0$, and

$$
\left(\chi_{\operatorname{supp} G_{q}}(x) u_{p q}(x), \bar{\phi}(x)\right)=\int_{\mathbb{R}^{m}}\left(\chi_{\operatorname{supp} G_{q}}(x) u_{p q}(x), \phi(x, \lambda)\right) d \nu_{x}(\lambda) \in \mathbb{R}
$$

for $x \in D_{p q}$. By the assumption, there exists $D_{0} \in \mathfrak{A}$ with $\mu\left(\Omega \backslash D_{0}\right)=0$ such that $\bar{\phi}(x), \phi(x, \lambda) \in \operatorname{vsupp} X(x)=\operatorname{vsupp} Y(x)$ for all $x \in D_{0}$ and for all $\lambda \in \mathbb{R}^{m}$. Hence, for $x \in D_{0} \cap \bigcap_{p=1}^{d(q)} D_{p q}$ and $1 \leq p \leq d(q)$, the integral $\int_{\mathbb{R}^{m}} \phi(x, \lambda) d \nu_{x}(\lambda)$ exists in the finite-dimensional Euclidean space $\operatorname{vsupp} Y(x)=\operatorname{vsupp} X(x)$ and

$$
\left(u_{p q}(x), \bar{\phi}(x)\right)=\left(u_{p q}(x), \int_{\mathbb{R}^{m}} \phi(x, \lambda) d \nu_{x}(\lambda)\right) \in \mathbb{R} .
$$


Therefore,

$$
\bar{\phi}(x)=\int_{\mathbb{R}^{m}} \phi(x, \lambda) d \nu_{x}(\lambda) \in \operatorname{vsupp} X(x)
$$

for $x \in D_{\phi}:=\bigcup_{q=1}^{\infty}\left[D_{0} \cap \bigcap_{p=1}^{d(q)} D_{p q}\right]$, and $\mu\left(\Omega \backslash D_{\phi}\right)=0$. Hence the statement (Y3) follows for $A_{\phi}:=\Omega \backslash D_{\phi}$.

STEP 3.2 (Proof of (Y4)). Observe that, as $X=Y^{\prime}$, there exist a subsequence $j(k)$ of $j_{k}$ and $\bar{\phi} \in X$ such that $\phi \circ z_{j(k)}$ is $Y$-weakly convergent to $\bar{\phi}$ in $X$, due to the $Y$-weak completeness theorem of J. Dieudonné [7] (if $X$ is a normed lattice with $\left.Y=X^{\prime}\right)$; W. Luxemburg and A. Zaanen [10], P. P. Zabrejko [23, Theorem 32] (if $X$ is a normed lattice); H. Nakano [13] $\left(d=1\right.$ with $\left.Y=X^{\prime}\right)$; O. Burkinshaw and P. Dodds [4, Corollary 4.2 of Theorem 4.1] $(d=1)$ and [15, Theorem 2.8/(1)], [18] $(d \geq 2)$.

By Theorem $2.1 /(\mathrm{Y} 3)$ applied to $\phi \circ z_{j(k)}$, we can find $A_{\phi} \in \mathfrak{A}$ such that $\mu\left(A_{\phi}\right)=0$ and the integral $\int_{\mathbb{R}^{m}} \phi(x, \lambda) d \nu_{x}(\lambda)$ exists in vsupp $X(x)$ for $x \in \Omega \backslash A_{\phi}$.

We proceed to show that $\phi \circ z_{j_{k}}$ is $Y$-weakly convergent to $\widetilde{\phi}$ in $X$, where $\widetilde{\phi}(x):=\int_{\mathbb{R}^{m}} \phi(x, \lambda) d \nu_{x}(\lambda)$ for $x \in \Omega \backslash A_{\phi}$ and $\widetilde{\phi}(x):=0$ otherwise.

On the contrary, suppose that $\phi \circ z_{j_{k}}$ is not $Y$-weakly convergent to $\widetilde{\phi}$ in $X$. Then there exist $\varepsilon>0, h_{0} \in Y$ and a subsequence $q_{k}$ of $j_{k}$ such that $\left|\left\langle\phi \circ z_{q_{k}}, h_{0}\right\rangle_{\mu}-\left\langle\widetilde{\phi}, h_{0}\right\rangle_{\mu}\right|>\varepsilon>0$. By the above $Y$-weak completeness theorem together with Theorem 2.1/(Y3), for the sequence $\phi \circ z_{q_{k}}$ we can find a subsequence $i_{k}$ of $q_{k}, \widehat{\phi} \in X$ and $A_{\widehat{\phi}} \in \mathfrak{A}$ such that $\left\langle\phi \circ z_{i_{k}}, h\right\rangle_{\mu} \rightarrow\langle\widehat{\phi}, h\rangle_{\mu}(\forall h \in Y)$, $\mu\left(A_{\widehat{\phi}}\right)=0$, the integral $\int_{\mathbb{R}^{m}} \phi(x, \lambda) d \nu_{x}(\lambda)$ exists in $\operatorname{vsupp} X(x)$ for $x \in$ $\Omega \backslash A_{\widehat{\phi}}$, and $\widehat{\phi}(x)=\int_{\mathbb{R}^{m}} \phi(x, \lambda) d \nu_{x}(\lambda)$ on $\Omega \backslash A_{\widehat{\phi}}$. Therefore, $\widehat{\phi}$ and $\widetilde{\phi}$ define the same element (equivalence class) in $X$, and $\left\langle\widehat{\phi}, h_{0}\right\rangle_{\mu}=\left\langle\widetilde{\phi}, h_{0}\right\rangle_{\mu}$. Hence, we get a contradiction.

Proposition 3.1 ([15, Lemma 4.2.2]). Let $\mu(\Omega)=\infty$. Then, for a sequence $z_{j} \in L^{0}\left(\mathbb{R}^{m}\right)$, the condition (LB) holds with respect to $\mu$ if and only if the condition (GB) holds with respect to $\mu_{*}$.

Proof of Theorem 2.3. By Proposition 3.1, (LB) for $\mu$ and $z_{j}$ implies (GB) for $\mu_{*}$ and $z_{j}$. So, we may apply Theorem 2.1 for $z_{j}$ with respect to $\mu_{*}$. Recall that if $\mu(\Omega)=\infty$ then the measure $\mu$ is called separable (see [9, 23]) provided $\mu_{*}$ is separable, which is equivalent to separability of $L^{0}\left(\mathbb{R}^{m}\right)$. We divide the proof into Steps 3.3-3.4.

Step 3.3 (Proof of (Y3)). Denote by $\alpha_{*} \in L^{1}((0, \infty))$ the Radon-Nikodym derivative $d \mu_{*} / d \mu$. Define

$$
\begin{aligned}
\widetilde{Y} & :=\left\{\tilde{z}^{\prime}: \alpha_{*} \tilde{z}^{\prime} \in Y\right\}, \\
\widetilde{Y}_{\mu_{*}}^{\prime} & :=\left\{z \in L^{0}\left(\mathbb{R}^{m}\right): z(x) \in \operatorname{vsupp} \widetilde{Y}(x) \mu_{*} \text {-a.e., }\left\langle z, \tilde{z}^{\prime}\right\rangle_{\mu_{*}} \in \mathbb{R}, \forall \tilde{z}^{\prime} \in \widetilde{Y}\right\},
\end{aligned}
$$


where $\left\langle z, \tilde{z}^{\prime}\right\rangle_{\mu_{*}}:=\int_{\Omega}\left(z(x), \tilde{z}^{\prime}(x)\right) d \mu_{*}(x)$. Then $\tilde{Y}_{\mu_{*}}^{\prime}$ is in fact the Köthe associate space of $\widetilde{Y}$ with respect to $\mu_{*}$. Observe that, for $\alpha_{*} \tilde{z}^{\prime}=z^{\prime} \in Y$,

$$
\left\langle z, \tilde{z}^{\prime}\right\rangle_{\mu_{*}}=\int_{\Omega}\left(z(x), z^{\prime}(x) / \alpha_{*}(x)\right) \alpha_{*}(x) d \mu(x)=\left\langle z, z^{\prime}\right\rangle_{\mu} .
$$

As $\zeta \in L^{1}\left(\Omega, C_{0}\left(\mathbb{R}^{m}\right) ; \mu\right)$ if and only if $\widetilde{\zeta}:=\zeta / \alpha_{*} \in L^{1}\left(\Omega, C_{0}\left(\mathbb{R}^{m}\right) ; \mu_{*}\right)$, we have

$$
\langle\nu, \widetilde{\zeta}\rangle_{\mu_{*}}:=\int_{\Omega}\left[\int_{\mathbb{R}^{m}} \widetilde{\zeta}(x, \lambda) d \nu_{x}(\lambda)\right] d \mu_{*}(x)=\langle\nu, \zeta\rangle_{\mu} .
$$

Hence, $\delta_{z_{j_{k}}}$ is $L^{1}\left(\Omega, C_{0}\left(\mathbb{R}^{m}\right) ; \mu_{*}\right)$ weakly convergent to $\nu$ in $L_{\omega}^{\infty}\left(\Omega, \mathcal{M}\left(\mathbb{R}^{m}\right) ; \mu_{*}\right)$ and $\phi \circ z_{j_{k}}$ is $\widetilde{Y}$-weakly convergent to $\bar{\phi}$ in $X$ with respect to the duality pair $\langle X, \tilde{Y}\rangle_{\mu_{*}}$. By Theorem $2.1 /(\mathrm{Y} 3)$, there exists $A_{\phi} \in \mathfrak{A}$ such that $\mu_{*}\left(A_{\phi}\right)=0$, the integral $\int_{\mathbb{R}^{m}} \phi(x, \lambda) d \nu_{x}(\lambda)$ exists in vsupp $X(x)$ for $x \in \Omega \backslash A_{\phi}$, and (2.1) holds for all $x \in \Omega \backslash A_{\phi}$. As $\mu$ is equivalent to $\mu_{*}$, we see that $\mu\left(A_{\phi}\right)=0$.

SteP 3.4 (Proof of (Y4)). Observe that $X=Y^{\prime}$ implies $X=\tilde{Y}_{\mu_{*}}^{\prime}$. Since the sequence $\phi \circ z_{j_{k}}$ is sequentially $Y$-weakly pre-compact in $X$, we conclude that $\phi \circ z_{j_{k}}$ is sequentially $\tilde{Y}$-weakly pre-compact in $X$ with respect to the duality pair $\langle X, \widetilde{Y}\rangle_{\mu_{*}}$. By Theorem $2.1 /(\mathrm{Y} 4)$, there exists $A_{\phi} \in \mathfrak{A}$ such that $\mu_{*}\left(A_{\phi}\right)=0$, the integral $\int_{\mathbb{R}^{m}} \phi(x, \lambda) d \nu_{x}(\lambda)$ exists in $\operatorname{vsupp} X(x)$ for all $x \in$ $\Omega \backslash A_{\phi}$, and $\phi \circ z_{j_{k}}$ is $\widetilde{Y}$-weakly convergent to $\widetilde{\phi}$ in $X$ with respect to $\langle\cdot, \cdot\rangle_{\mu_{*}}$, where $\widetilde{\phi}(x):=\int_{\mathbb{R}^{m}} \phi(x, \lambda) d \nu_{x}(\lambda)$ for $x \in \Omega \backslash A_{\phi}$ and $\widetilde{\phi}(x):=0$ otherwise. Since $\mu$ is equivalent to $\mu_{*}$, we conclude that $\mu\left(A_{\phi}\right)=0$ and $\phi \circ z_{j_{k}}$ is $Y$-weakly convergent to $\widetilde{\phi}$ in $X$.

Proof of Proposition 2.4. We divide this proof into Steps 3.5-3.6.

STEP 3.5. Assume that (SC1) holds. We claim that the sequence $\phi \circ z_{j}$ is $Y$-absolutely bounded in $X$, i.e.

$$
\begin{array}{r}
y \in Y \Rightarrow \lim _{\mu_{*}(D) \rightarrow 0} \sup _{j \in \mathbb{N}} \int_{D}\left|\left(y(x),\left(\phi \circ z_{j}\right)(x)\right)\right| d \mu(x)=0, \\
\quad \sup _{j \in \mathbb{N}} \int_{\Omega}\left|\left(y(x),\left(\phi \circ z_{j}\right)(x)\right)\right| d \mu(x)<\infty .
\end{array}
$$

Indeed, we deduce that

$$
\begin{aligned}
\int_{D}\left|\left(y(x),\left(\phi \circ z_{j}\right)(x)\right)\right| d \mu(x) \leq \int_{D}|y(x)| \gamma\left(\left|z_{j}(x)\right|\right) u_{0}(x) d \mu(x) \\
=\left(\int_{D \cap\left\{\gamma\left(\left|z_{j}(\cdot)\right|\right) \leq l\right\}}+\int_{D \cap\left\{\gamma\left(\left|z_{j}(\cdot)\right|\right) \geq l\right\}}\right)|y(x)| \gamma\left(\left|z_{j}(x)\right|\right) u_{0}(x) d \mu(x) \\
\quad \leq l \int_{D}|y(x)| u_{0}(x) d \mu(x)+\int_{\left\{\gamma\left(\left|z_{j}(\cdot)\right|\right) \geq l\right\}}|y(x)| \gamma\left(\left|z_{j}(x)\right|\right) u_{0}(x) d \mu(x) .
\end{aligned}
$$


Since $\gamma$ is nondecreasing, we can choose $m_{l} \rightarrow \infty$ such that $\{t \geq 0: \gamma(t) \geq l\}$ $\subset\left\{t \geq 0: t \geq m_{l}\right\}$. Then

$$
\begin{aligned}
\int_{\left\{\gamma\left(\left|z_{j}(\cdot)\right|\right) \geq l\right\}}|y(x)| \gamma\left(\left|z_{j}(x)\right|\right) u_{0}(x) d \mu(x) \\
\leq \int_{\left\{\left|z_{j}(\cdot)\right| \geq m_{l}\right\}}|y(x)| \gamma\left(\left|z_{j}(x)\right|\right) u_{0}(x) d \mu(x) \\
\leq \frac{1}{M_{l}} \int_{\left\{\left|z_{j}(\cdot)\right| \geq m_{l}\right\}}|y(x)| g\left(\left|z_{j}(x)\right|\right) u_{0}(x) d \mu(x) \\
\leq \frac{1}{M_{l}} \int_{\Omega}|y(x)| g\left(\left|z_{j}(x)\right|\right) u_{0}(x) d \mu(x) \leq \frac{C}{M_{l}} \rightarrow 0
\end{aligned}
$$

as $l \rightarrow \infty$ uniformly in $j$, where $C \in(0, \infty), g(t) \geq M_{l} \gamma(t)$ for $t \geq m_{l}$, and $M_{l} \rightarrow \infty$ as $l \rightarrow \infty$. Hence, for any $\varepsilon>0$ there exists $l_{0}$ such that

$$
\int_{\left\{\gamma\left(\left|z_{j}(\cdot)\right|\right) \geq l_{0}\right\}}|y(x)| \gamma\left(\left|z_{j}(x)\right|\right) u_{0}(x) d \mu(x) \leq \varepsilon \quad \forall j \in \mathbb{N} .
$$

As $y \in Y$ and $u_{0} Y \subset L^{1}\left(\mathbb{R}^{d}\right)$ we have $\lim _{\mu_{*}(D) \rightarrow 0} \int_{D}|y(x)| u_{0}(x) d \mu(x)$ $=0$. Therefore, there exists $\delta>0$ such that $\mu_{*}(D)<\delta$ implies

$$
\int_{D}\left|y(x) u_{0}(x)\right| d \mu(x) \leq \frac{\varepsilon}{l_{0}} .
$$

Hence, we infer that

$$
\mu_{*}(D)<\delta \Rightarrow \int_{D}\left|\left(y(x),\left(\phi \circ z_{j}\right)(x)\right)\right| d \mu(x) \leq l_{0} \frac{\varepsilon}{l_{0}}+\varepsilon=2 \varepsilon .
$$

So, the first part of (3.2) follows. The second part of (3.2) follows by the same arguments.

Since $\operatorname{vsupp} X(x)=\operatorname{vsupp} Y(x) \mu$-a.e. and $X \subset Y^{\prime}$, (3.2) implies that the sequence $\phi \circ z_{j_{k}}$ is sequentially $Y$-weakly pre-compact in $X$, due to the $Y$-weak pre-compactness theorem of J. Dieudonné [7] (if $X$ is a normed lattice with $X=X^{\prime \prime}, Y=X^{\prime}$ ); W. Luxemburg and A. Zaanen [10], P. P. Zabrejko [23, Theorem 33] (if $X$ is a normed lattice); H. Nakano [13] $(m=1$ with $X=X^{\prime \prime}, Y=X^{\prime}$ ); O. Burkinshaw and P. Dodds [4, Theorem 3.4, Proposition 2.4] $(m=1)$, and [15, Theorem 2.8/(2)], [18] $(m \geq 2)$.

STEP 3.6. Assume that (SC2) holds. It is known that $\left(\Gamma^{\circ}\right)^{\prime}$ can be interpreted as the dual space $\left(\Gamma^{\circ}\right)^{*}$ by the injection $z^{\prime} \mapsto\left\langle\cdot, z^{\prime}\right\rangle_{\mu}$ (see, e.g., [1, 23], [9, Theorems VI.1.4 and IV.3.6] $(d=1)$, [15, Corollary 2.2, Proposition 2.2], [18] $(d \geq 2))$. By [9, Theorem IV.3.3] $(m=1)$ and [16, Theorem 2.5], [15, 18] $(m \geq 2)$, the separability of $\mu$ implies the separability of $\Gamma^{\circ}$. Hence, by the Alaoglu-Bourbaki theorem together with [9, Theorem V.7.6], the $\Gamma^{\circ}$-weak 
topology on any closed ball of $\left(\Gamma^{\circ}\right)^{*}$ is compact and metrizable. Therefore, for any sequence $a_{i}$ in the $\left(\Gamma^{\circ}\right)^{\prime}$-norm-bounded set $\left\{\phi \circ z_{j_{k}}\right\}_{k \in \mathbb{N}}$ there exist a subsequence $p(i)$ of the sequence $i$ and $a \in\left(\Gamma^{\circ}\right)^{\prime}$ such that $a_{p(i)}$ is $\Gamma^{\circ}$-weakly convergent to $a$ in $\left(\Gamma^{\circ}\right)^{\prime}$. Since $Y \subset \Gamma^{\circ}$ and $\left(\Gamma^{\circ}\right)^{\prime} \subset X, a_{p(i)}$ is $Y$-weakly convergent to $a$ in $X$. Hence, $a_{p(i)}$ is a $Y$-weak Cauchy sequence in $X$. Thus, the statement of Proposition 2.4/(SC2) follows.

Proof of Theorem 2.6. It suffices to modify Step 3.1 of the proof of Theorem 2.1/(Y3). Since supp $\widetilde{K}=\Omega$, by [9, Corollary IV.3.2] there exists a sequence of disjoint sets $\Omega_{q} \in \mathfrak{A}$ such that $\chi_{\Omega_{q}} \in \widetilde{K}$ and $\mu\left(\Omega \backslash \bigcup_{q=1}^{\infty} \Omega_{q}\right)=0$. Since $E$ is a separable reflexive space, so is $E^{*}$. Hence, there exists $\left\{\widetilde{u}_{p}\right\}_{p \in \mathbb{N}}$ dense in $E^{*}$. By (3.1) for $\chi_{\Omega_{q}} \widetilde{u}_{p} \in Y$, for some $\widetilde{D}_{p q} \in \mathfrak{A}, \widetilde{D}_{p q} \subset \Omega_{q}$ and $\mu\left(\Omega_{q} \backslash \widetilde{D}_{p q}\right)=0$ and $\left\langle\chi_{\Omega_{q}}(x) \widetilde{u}_{p}, \bar{\phi}(x)\right\rangle=\int_{\mathbb{R}^{m}}\left\langle\chi_{\Omega_{q}}(x) \widetilde{u}_{p}, \phi(x, \lambda)\right\rangle d \nu_{x}(\lambda) \in \mathbb{R}$ for $x \in \widetilde{D}_{p q}$. Therefore, for $x \in \bigcap_{p \in \mathbb{N}} \widetilde{D}_{p q}$,

$$
\left\langle\widetilde{u}_{p}, \bar{\phi}(x)\right\rangle=\int_{\mathbb{R}^{m}}\left\langle\widetilde{u}_{p}, \phi(x, \lambda)\right\rangle d \nu_{x}(\lambda) \in \mathbb{R} .
$$

Put $\psi(x, \lambda):=\|\phi(x, \lambda)\|_{E}$. Since $\operatorname{supp} K=\operatorname{supp} \widetilde{K}=\Omega$ and the sequence $\phi \circ z_{j_{k}}$ is $\widetilde{K}\left(E^{*}\right)$-weakly pre-compact in $K(E)$, by M. Talagrand [22, Corollary 9 of Theorem 6] and M. Nowak [19, Theorem 3.3] we deduce that the sequence $\psi \circ z_{j_{k}}$ is $\widetilde{K}$-weakly pre-compact in $\widetilde{K}^{\prime}$. By Theorem $2.1 /(\mathrm{Y} 4)$ for $\psi \circ z_{j_{k}}$, there exists $D_{\psi} \in \mathfrak{A}$ such that $\mu\left(\Omega \backslash D_{\psi}\right)=0$ and the integral $\int_{\mathbb{R}^{m}} \psi(x, \lambda) d \nu_{x}(\lambda) \in$ $\mathbb{R}$ exists for all $x \in D_{\psi}$.

Fix $u^{*} \in E^{*}$. Then we can choose a sequence $\hat{u}_{i}:=\widetilde{u}_{p(i)}$ from the dense set $\left\{\widetilde{u}_{p}\right\}_{p \in \mathbb{N}}$ with $\left\|\hat{u}_{i}-u^{*}\right\|_{E^{*}} \rightarrow 0$ as $i \rightarrow \infty$. Hence, $x \in \bigcap_{p=1}^{\infty} \widetilde{D}_{p q} \cap D_{\psi}$ implies that $\left\langle\hat{u}_{i}, \phi(x, \lambda)\right\rangle \rightarrow\left\langle u^{*}, \phi(x, \lambda)\right\rangle$ for all $\lambda \in \mathbb{R}^{m},\left\langle\hat{u}_{i}, \bar{\phi}(x)\right\rangle \rightarrow\left\langle u^{*}, \bar{\phi}(x)\right\rangle$, and $\left|\left\langle\hat{u}_{i}, \phi(x, \lambda)\right\rangle\right| \leq \sup _{i \in \mathbb{N}}\left\|\hat{u}_{i}\right\|_{E^{*}} \psi(x, \lambda)<\infty$. Hence, by the Lebesgue dominated convergence theorem, we infer that

$$
\int_{\mathbb{R}^{m}}\left\langle\hat{u}_{i}, \phi(x, \lambda)\right\rangle d \nu_{x}(\lambda) \rightarrow \int_{\mathbb{R}^{m}}\left\langle u^{*}, \phi(x, \lambda)\right\rangle d \nu_{x}(\lambda) \in \mathbb{R}
$$

as $i \rightarrow \infty$ for $x \in \bigcap_{p=1}^{\infty} \widetilde{D}_{p q} \cap D_{\psi}$. Hence, $x \in \bigcap_{p=1}^{\infty} \widetilde{D}_{p q} \cap D_{\psi}$ implies that $\left\langle u^{*}, \bar{\phi}(x)\right\rangle=\int_{\mathbb{R}^{m}}\left\langle u^{*}, \phi(x, \lambda)\right\rangle d \nu_{x}(\lambda) \in \mathbb{R}$ for all $u^{*} \in E^{*}$. Therefore, for $x \in \bigcap_{p=1}^{\infty} \widetilde{D}_{p q} \cap D_{\psi}$, the Pettis integral $(P)-\int_{\mathbb{R}^{m}} \phi(x, \lambda) d \nu_{x}(\lambda) \in E$ exists and coincides with $\bar{\phi}(x)[6$, p. 53]. So, we obtain

$$
\left(x \in D_{\phi}:=\bigcup_{q=1}^{\infty} \bigcap_{p=1}^{\infty} \widetilde{D}_{p q} \cap D_{\psi}\right) \Rightarrow \bar{\phi}(x)=(P)-\int_{\mathbb{R}^{m}} \phi(x, \lambda) d \nu_{x}(\lambda) \in E,
$$

and $\mu\left(\Omega \backslash D_{\phi}\right)=0$. Hence, the statement (Y3) of Theorem 2.6 follows for $A_{\phi}:=\Omega \backslash D_{\phi}$. 
Lemma 3.2 ([15, Lemma 4.2.3]). Let $K \subset L^{0}(\mathbb{R})$ be a normed lattice with monotone norm. Then for $\varepsilon \in(0, \infty)$ there exists $r(\varepsilon) \in(0, \infty)$ such that $\|z\|_{K} \leq r(\varepsilon) \Rightarrow\|z\|_{L^{0}(\mathbb{R})} \leq \varepsilon$.

Proof of Proposition 2.7. By 2.7, $g_{q}\left(\left|z_{j}\left(x_{0}\right)\right|\right) \geq g_{q}(L)$ for $x_{0} \in D_{L}^{j}:=$ $\left\{x \in \Omega:\left|z_{j}(x)\right| \geq L\right\}$. Since $K(q)$ is a normed lattice with monotone norm $\|\cdot\|_{K(q)}$, we infer that

$$
\begin{aligned}
\left\|\chi_{C_{q}} g_{q}\left(\left|z_{j}(\cdot)\right|\right)\right\|_{K(q)} & \geq\left\|\chi_{C_{q} \cap D_{L}^{j}} g_{q}\left(\left|z_{j}(\cdot)\right|\right)\right\|_{K(q)} \\
& \geq\left\|\chi_{C_{q} \cap D_{L}^{j}} g_{q}(L)\right\|_{K(q)}=g_{q}(L)\left\|\chi_{C_{q} \cap D_{L}^{j}}\right\|_{K(q)} .
\end{aligned}
$$

Hence, $\lim _{L \rightarrow \infty} \sup _{j \in \mathbb{N}}\left\|\chi_{C_{q} \cap D_{L}^{j}}\right\|_{K(q)}=0$. By Lemma 3.2, for all $\varepsilon>0$ there exists $r_{q}(\varepsilon)>0$ such that, given $j \in \mathbb{N}$, if $\left\|\chi_{C_{q} \cap D_{L}^{j}}\right\|_{K(q)} \leq r(\varepsilon)$ then $\left\|\chi_{C_{q} \cap D_{L}^{j}}\right\|_{L^{0}(\Omega, \mathbb{R})}=\frac{1}{2} \mu_{*}\left(C_{q} \cap D_{L}^{j}\right) \leq \varepsilon$. Therefore, there exists $L_{\varepsilon}^{q}$ such that $L \geq L_{\varepsilon}^{q}$ implies that $\left\|\chi_{C_{q} \cap D_{L}^{j}}\right\|_{K(q)} \leq r(\varepsilon)$ for all $j \in \mathbb{N}$. It follows that $\frac{1}{2} \mu_{*}\left(C_{q} \cap D_{L}^{j}\right) \leq \varepsilon$ for all $j \in \mathbb{N}$ and all $L \geq L_{\varepsilon}^{q}$. This gives (GB) for $\mu_{*}$ and $z_{j}$ on $C_{q} \subset \Omega$. By Proposition 3.1, (LB) follows for $\mu$ and $z_{j}$.

Acknowledgements. We are grateful to the referee for many constructive remarks used for preparing the revised version.

\section{References}

[1] J. Appell and P. P. Zabrejko, Nonlinear Superposition Operators, Cambridge Univ. Press, Cambridge, 1990.

[2] E. J. Balder, A general approach to lower semicontinuity and lower closure in optimal control theory, SIAM J. Control Optim. 22 (1984), 570-598.

[3] J. M. Ball, A version of the fundamental theorem for Young measures, in: PDE's and Continuum Models of Phase Transitions (Nice, 1988), D. Serre et al. (eds.), Lecture Notes in Phys. 344, Springer, Berlin, 1989, 207-215.

[4] O. Burkinshaw and P. Dodds, Weak sequential compactness and completeness in Riesz spaces, Canad. J. Math. 28 (1976), 1332-1339.

[5] C. Castaing, P. Raynaud de Fitte, and M. Valadier, Young Measures on Topological Spaces with Applications in Control Theory and Probability Theory, Kluwer, Dordrecht, 2004.

[6] J. Diestel and J. J. Uhl, Vector Measures, Amer. Math. Soc., Providence, 1977.

[7] J. Dieudonné, Sur les espaces de Köthe, J. Anal. Math. 1 (1951), 81-115.

[8] I. Fonseca and G. Leoni, Modern Methods in the Calculus of Variations: $L^{p}$ Spaces, Vol. I, Springer, Berlin, 2007.

[9] L. V. Kantorovich and G. P. Akilov, Functional Analysis, Pergamon Press, Oxford, 1982.

[10] W. A. J. Luxemburg and A. C. Zaanen, Compactness of integral operators in Banach function spaces, Math. Ann. 149 (1963), 150-180.

[11] J. Málek, J. Nečas, M. Rokyta, and M. Růžička, Weak and Measure-Valued Solutions to Evolutionary PDEs, Chapman \& Hall, London, 1996. 
[12] J. Musielak, Orlicz Spaces and Modular Spaces, Lecture Notes in Math. 1034, Springer, Berlin, 1983.

[13] H. Nakano, Modulared Semi-Ordered Linear Spaces, Maruzen, Tokyo, 1950.

[14] H. T. Nguyêñ, Theory of semimodules of infra-semi-units in vector functions ideal spaces and its applications to integral operators, Dokl. Akad. Nauk SSSR 317 (1991), 1303-1307 (in Russian); English transl.: Soviet Math. Dokl. 43 (1991), 615-619.

[15] -, Non-Solid Ideal Spaces of Vector Functions: Geometry, Interpolation and Applications to Nonlinear Operators and Equations, Habilitation dissertation, Belarus State Univ., Minsk, 1991, 341 pp.

[16] -, On Banach function M-spaces, Indag. Math. (N.S.) 14 (2002), 551-564.

[17] H. T. Nguyêñ and D. Pączka, Generalized gradients for locally Lipschitz integral functionals on non- $L^{p}$-type spaces of measurable functions, in: Function Spaces VIII, H. Hudzik et al. (eds.), Banach Center Publ. 79, Inst. Math., Polish Acad. Sci., 2008, $135-156$.

[18] H. T. Nguyêñ and P. P. Zabrejko, Ideal spaces of vector-valued functions and their applications, in: Function Spaces 1989, J. Musielak et al. (eds.), Teubner-Texte Math. 120, Teubner, Stuttgart-Leipzig, 1991, 112-119.

[19] M. Nowak, Conditional and relative weak compactness in vector-valued function spaces, J. Convex Anal. 12 (2005), 447-463.

[20] P. Pedregal, Parametrized Measures and Variational Principles, Birkhäuser, Basel, 1997.

[21] T. Roubiček, Relaxation in Opimization Theory and Variational Calculus, de Gruyter, Berlin, 1997.

[22] M. Talagrand, Weak Cauchy sequences in $L^{1}(E)$, Amer. J. Math. 106 (1984), 703727.

[23] P. P. Zabrejko, Ideal spaces of functions I, Vestnik Yaroslav. Univ. 8 (1974), 12-52.

Hôǹg Thái Nguyêñ

Institute of Mathematics

Szczecin University

Wielkopolska 15

70-451 Szczecin, Poland

E-mail: nguyenhthaimathuspl@yahoo.com
Dariusz Pączka Institute of Mathematics Szczecin University of Technology

Al. Piastów 48 70-311 Szczecin, Poland E-mail: paczka@ps.pl

Received October 3, 2007;

received in final form July 23, 2008 